\title{
Overcoming the challenge of back pain complaints
}

\author{
Bruce Rothschild* \\ West Virginia University, School of Medicine, Morgantown, WV 26501
}

\begin{abstract}
Back pain is one of the more challenging aspects of medical care, for which many physicians feel unprepared. The dichotomy between mechanical and inflammatory derivation is blurred and the possibility of referred pain is always present. Solutions are facilitated with careful localization and identification of exacerbating and ameliorating factors. Fibromyalgia, pain reproducible by pressure on specific points, must be distinguished from general body pain. It is the clinical evaluation that allows distinguishing among the most common causes of back pain and assures minimal chance of overlooking nonmusculoskeletal explanations. Removal of narcotics from the therapeutic armamentarium is a good start. Pain is often accompanied by depression, although common measures of depression are not specific, being a component of the pain itself. Mechanical solutions to back pain, whether perceived as mechanical or inflammatory in derivation, are reasonable initial approaches. Finally, the importance of sleep hygiene cannot be overemphasized, as disturbed sleep aggravates pain, even if it is not the initiating factor. This article addresses back pain amenability not only to clinical diagnosis but also to effective intervention.
\end{abstract}

Keywords: back pain, clinical evaluation, fibromyalgia, MRI, sleep, spondyloarthropathy.

\section{Introduction}

\section{Back pain as the clinician's nemesis}

Back pain has long been perceived as the physician's nemesis. Indeed, Osler [1] is credited with the admonition that physician's offices should have two doors: One for the patient to enter and one allowing the physician to exit when the patient's problem has been identified as back pain. One challenge has been categorizing the pain as mechanical or inflammatory, assuming that the causes are not mutually exclusive and that characterizations are reproducible without overlap. Activity-aggravated pain, reduced with rest, has been considered classic for mechanical back disorders, while worsening of back pain with rest and presence of morning stiffness of greater than one hour's duration have been considered indicative of inflammatory derivation of the pain [2]. The latter is often also characteristic of fibromyalgia, a disorder of unclear derivation, but not considered either inflammatory or mechanical in nature.

\section{Inflammation-derived back pain}

The major disorder responsible for inflammatory back pain is spondyloarthropathy in its several forms [2]: Ankylosing spondylitis, psoriatic arthritis, reactive arthritis, the arthritis of inflammatory bowel disease and an undifferentiated form. These are considered autoimmune disorders in which the body fails to recognize itself and reacts as if it is being attacked by a foreign invader. There are several genetic predispositions (e.g., HLA-B27), but these markers are also present in apparently healthy individuals. One form of spondyloarthropathy, reactive arthritis, represents a reaction to an inter-current event, infection agent (e.g., Salmonella) diarrhea, also referred to as food poisoning.

\section{Referred back pain}

Referred pain, that radiating in various defined patterns, has been considered evidence for nerve root impingement, yet also occurs in fibromyalgia [2, 3]. It is essential to consider as it such a common cause of back pain and a diagnosis so often overlooked. The latter has reproducible pain "radiation" distribution, although patterns do not necessarily follow traditionally recognized nerve pathways. Curiously, those patterns emulate acupuncture meridian, a pathophysiology unfamiliar to this western practitioner. Pain patterns and other complaints in fibromyalgia often elude explanation predicated on western concepts of medicine. However, a physical basis for patient complaints is evidenced by their disappearance when total resolution of the other symptoms of fibromyalgia 
is achieved. This resolution is not just of pain, but also of associated phenomena (e.g., irritable bowel syndrome, headaches).

\section{Technician versus clinician}

Physician frustration is understandable as we are taught to treat identified problems, using evidence-based approaches and identification of the source of back pain (the problem) has traditionally proven elusive to investigation by standard tools. This has led some orthopedic surgeons to require a MRI before they will even schedule a patient with back pain. There perhaps is a logic to that approach as they are people of action, seeking to identify an actionable (at least surgically) problem. Just as many physicians (that some call serologists, rather than clinicians) have turned to blood tests as the first investigative approach for patients with musculoskeletal pain in general, so too has MRI been pursued at the expense of in depth clinical assessment. Sacroiliac joint findings on radiologic evaluation (e.g., MRI) have been considered a major diagnostic indicator for presence of spondyloarthropathy [2]. However, the limited sensitivity and specificity of all radiologic modalities (including MRI) is insufficient for confident diagnosis (see below).

\section{Clinical investigation of back pain}

I suggest reemphasis on cultivation of clinical skills, despite their being less cost/time effective than for the physician to order tests, be they serologic or radiologic. Can the clinical evaluation be more efficient? Consider the following:

1. Localization. Have the patient point to where it hurts. Identification of lateral pelvic pain suggests involvement of one of the bursae (gluteus medius, gluteus minimus, subgluteus medius and subgluteus minimus) surrounding the greater trochanter. Tail bone localization suggests possible ischial bursitis. Actual low back localization is less diagnostically significant. The "it hurts everywhere" complaint is easier to diagnose when it is assured that the patient is reporting pain in each potential area (e.g., named joints/ muscles/ tendon insertions), requesting that the patient point to the pain's actual location(s) rather than depend upon the patient's terminology with all the latter's associated semantic variation. Patient's often use the term "hip" to report pain that is actually localized to what a physician would recognize (on pointing) as buttock, lateral pelvis and even low back.

2. Referred or radiation of pain. It is of course essential to distinguish a contiguous course of pain from that involving multiple areas in a perceived distribution. Pain radiating down a leg may reflect nerve distribution or simply pain in the back, knee and foot, occurring simultaneously, but unconnected. The distribution provides insights which may direct assessment beyond that of routine approaches to physical examination.

3. Exacerbation. Pain worse with activity, reduced with rest, worse during the work week and relieved by work "holidays" is of course highly suggestive of its mechanical or postural derivation. The postural nature of work and exacerbation activities requires review. Of course, avocational activities may influence or even cause back pain, so full review of activities is important. One often elusive and controversial diagnosis is that of facet disease, yet pain exacerbation on retroflexion is highly suggestive. The general extensive review of systems is helpful. Nonmusculoskeletal causes of back pain are rare but often identifiable when the patient's full review reveals vascular symptoms and thrombotic events accompanying aneurysms, fatty food ingestion aggravation related to gall bladder disease, food effects related to ulcers, dysuria related to kidney infections, hematuria related to nephrolithiasis and weight loss related to cancer. This is in addition the fever that often accompanies infection.

\section{General body pain}

Perhaps the most frustrating clinical complaint (for all involved- patients, people they interact with and the physicians involved in their care) is general body pain. The patient hurts everywhere. Of course, it is important to review specific locations. Patients may refer to pain in multiple joints as global pain. This may be a sign of systemic arthritis and needs to be distinguished from muscle pain and from actual generalized pain. The latter is often the description given by patients with fibromyalgia, while clarification limits the distribution axially. However, patients with fibromyalgia often have a referred pattern, which may be reported as quite generalized, but is reproduced/exacerbated by pressure on pertinent trigger points (but not at neutral areas). Trigger points are well-defined anatomical locations at which application of pressure reproduces the patient's symptomatology [2]. This is exemplified by the surgeon with low back pain, radiating to his thigh, who 
reported pain relief with long operating room (OR) sessions. History revealed that he kept his wallet in his back pocket (except during surgery)-directly over a fibromyalgia trigger point. Wallectomy (not just closeting his wallet during OR sessions) was the solution and eliminated his pain.

There are perhaps three major diagnostic issues related to general body pain:

1. Fibromyalgia has been a controversial subject, compromised to a great extent by alternative diagnostic approaches. Originally identified by a reproducible pattern of tender or trigger points, and overlapping with the myofascial pain syndrome named by physiatrists, the name fibromyalgia has been applied to regional pain complaints, independent of trigger point detection. It is unclear that such application of the term is helpful, especially in distinguishing patients with what might be referred to as general body tenderness. Patients with classic fibromyalgia have disruption of rapid eye movement (REM) and stage 4 sleep, restoration of which often reduces or eliminates symptoms $[2,4]$.

Unfortunately narcotics interfere with accessing those sleep states and actually compromise any benefit that might be achieved with stage IV enhancing hypnotics (i.e., ambien, restoril, lunesta) intervention $[4,5]$. Injection of trigger points with lidocaine or electroacupuncture applied to these points (which incidentally represent acupuncture meridian) is often effective with patients with fibromyalgia, but not those with general body tenderness. The latter comprises a group of individuals who essentially perceive touch sensation as pain, recognized as general body tenderness or mechanical allodyna [6, 7]. Reducing pain perception with narcotics or analgesics has no significant benefit. Traditional approaches are ineffective. Narcotics only "dull" the individual, not the pain. It is unknown if psychotherapy or hypnosis will impact those patients, but the clinical solution would seem to require altering perception.

Notation of alteration of immune modulator levels in depressed individuals afflicted with chronic back pain may also afford an opportunity for innovative therapeutic approaches. Tumor necrosis factor alpha (TNF $\alpha)$ and interleukin six (IL-6) levels are elevated in depression [8] and correlate with reduced therapeutic responsiveness, psychological well-being and quality of life [9], while interleukin 17 (IL-17) levels correlate with anxiety [10] and neuropathic pain [11].

2. Depression is a controversial aspect of fibromyalgia, a subject confounded as the criteria typically used for diagnosis of depression are also inherent components of pain itself. While narcotics dull mental function and antidepressants have their own set of risks, an intriguing suggestion has been use of ketamine [12]. It is unclear if the reduction of level of depression is related to reduced pain or to the high related to its use, that high potentially initiating subsequent substance abuse [13].

3. A diagnostic challenge may be individuals with an agenda, whether for disability or as narcotic seeking behavior. There latter can be avoided by proscribing narcotics. There seems to be no evidence that they are more effective than nonnarcotic analgesics and actually compromise symptom resolution. Disability-seeking behavior can be recognized by full visit surveillance (from car to office) and reproducibility of findings. This is especially recognizable for fibromyalgia diagnoses, as two point sensory discrimination is insufficient to distinguish trigger and neutral points in the back [3]. Thus, reproducibility of pain response and lack of pain initiation by pressure at neutral points allow confident diagnosis of fibromyalgia, while inability to distinguish pain production at trigger and neutral points suggests either general body pain or a patient agenda.

\section{Diagnosis of spondyloarthropathy}

The challenge is no less for what has been categorized as inflammatory back pain, that related to ankylosing spondylitis, a subset of a larger set of related disorders referred to as spondyloarthropathy. Stereotypically perceived as back pain worsened with rest, improved with activity, associated with morning stiffness, limited back motion, radiologic evidence of sacroiliitis and a positive lab test- presence of HLA-B27. Unfortunately, that image compromises its accurate recognition. While HLA-B27 is present in 50-90\% of individuals with back involvement (not all individuals with spondyloarthropathy have axial joint disease), one in seven Caucasians and one in twenty five African Americans also are positive for HLA-B27. Given the population prevalence of spondyloarthropathy, 98 out 
of every 100 Caucasians with HLA-B27 are actually unafflicted by that disease $[14,15]$ ! So, such serologic diagnosis cannot be supported. Radiologic evidence of sacroiliitis seems an obvious indicator, but can it be "trusted"? The non-planar nature of the sacroiliac joint led to recommendation of a variety of x-ray positioning. Unfortunately, that proved problematic. A study of skeletons revealed apparent fusion of the sacroiliac joints when the components were freely movable and fused joints often did not so appear on $\mathrm{x}$ ray [16]. The same study revealed similar reliability problems with computed tomography and magnetic resonance imaging has also proven problematic. Periarticular edema proved to also be an unreliable indicator of the inflammatory involvement understood to be the primary pathophysiology of sacroiliitis [17]. Radiologic modalities (including MRI) seem to have insufficient resolution for confident recognition of sacroiliac joint disease.

Thus, we are left with diagnosis predicated on clinical skills. In the absence of accompanying peripheral joint disease, perhaps limited back motion is the major diagnostic clue. The morning stiffness of greater than an hour's duration, so characteristic of spondyloarthropathy, is also common in individuals with fibromyalgia. So it does not have unequivocal diagnostic significance. Assessment of forward flexion (Schöber test), lateral bending and flexion deformity (Flech test) are most diagnostic for spondyloarthropathy.

Spondyloarthropathy is considered an inflammatory disorder, but it is difficult to separate inflammation from mechanical sources of pain. Back pain in individuals with spondyloarthropathy may not be totally inflammatory in derivation. Pain persists in those with the ankylosing spondylitis variety until total fusion occurs, precluding spinal motion. It is suggested that the mechanical disadvantage produced by the abnormal posture (propensity to flexion deformity) of the disease stresses back muscles, leading to fatigue and pain. Anti-inflammatory and even biologic agents (e.g., tumor necrosis factor inhibitors) have been used to treat such pain, yet a mechanical approach is often also as effective: Back extension exercises appear to relieve related pain. However, all benefits of such a program are apparently precluded if flexion activities are not proscribed and avoided.

\section{Summary}

It is the clinical evaluation that allows distinguishing among the most common causes of back pain and assures minimal chance of overlooking nonmusculoskeletal explanations. Independent of one's attitude related to the diagnosing fibromyalgia, there seems to be little risk to recognizing and treating sleep disturbance. After all, sleep disturbance is one of the least generally addressed compromises of general health and function [5] and a good night's sleep (REM and stage IV) made such a difference in our training days. Introducing back exercises, whether conditioning for mechanical back pain or extension with avoidance of flexion activities for inflammatory disease, is costeffective and may eliminate need for analgesic approaches. Over the years, several non-steroidal antiinflammatory agents have proven as effective as narcotics [18], so one consideration is total avoidance of narcotic prescription. One can thus avoid the complications (both medical and legal) of their use and concentrate on what is actually responsible for the patient's complaints.

Related to the latter, it is essential to distinguish what has been referred to as engrams (brain patterns related to previous experience) from ongoing pain production. Sometimes longstanding pain results in continued brain perception/memory, even if the responsible physiologic process itself is no longer present. Such engrams and other misperceptions are not amenable to pain medication and require modification (e.g., behavioral) approaches. We all want to reduce pain and suffering and improve the quality of life for the patients we serve. It is our clinical skills which allow us to be successful in that endeavor.

\section{Conflicts of interest}

The author declares no conflicts of interest.

\section{References}

1. Osler W. The Principles and Practice of Medicine. Appleton Press, New York, USA, 1892.

2. Rothschild BM. Rheumatology: A Primary Care Approach. Yorke Medical Press, New York, USA, 1982.

3. Rothschild BM. Fibromyalgia:
Can one distinguish it from simulation? J Rheum 2001; 28: 2762-3.

4. Rothschild BM. Zolpidem efficacy in fibromyalgia. $\boldsymbol{J}$ Rheum 1997; 24: 1012.

5. Rothschild BM. To sleep, perchance to dream Restoration of normal sleep physiology versus sedation in management of sleep disorders and fibromyalgia. Rheumatologist 2015; 9(6): 67.

6. Abraira VE, Ginty DD. The sensory neurons of touch. Neuron 2013; 79: 618-39. doi: 10.1016/j.neuron.2013.07.051. 
7. Piers C, Seal RP. Neural circuits for pain: Recent advances and current views. Science 2016; 354 578-84. doi: 10.1126/science. aaf8933.

8. Liu Y, Ho RC, Mak A Interleukin (IL)-6, tumour necrosis factor alpha (TNF- $\alpha$ ) and soluble interleukin-2 receptors (sIL-2R) are elevated in patients with major depressive disorder: a meta-analysis and metaregression. J Affect Dis 2012; 139: 230-9. doi: 10.1016/j.jad. 2011.08.003. PMID:21872339.

9. Lasselin J, Kemani MK, Kanstrup M, Olsson GL, Axelsson J, Andreasson A, et al. Low-grade inflammation may moderate the effect of behavioral treatment for chronic pain in adults. $\boldsymbol{J}$ Behav Med 2016; 39: 916-24. doi: 10.1007/s10865-016-9769-z. PMID: 27469518.

10. Liu Y, Ho RC, Mak. The role of interleukin (IL)-17 in anxiety and depression of patients with rheumatoid arthritis. Intl $\boldsymbol{J}$ Rheum Dis 2012; 15: 183-7. PMID: 22462422, doi: 10.1111/j. 1756-185x.2011.01673.x.

11. Sun C, Zhang J, Chen L, Liu T, $\mathrm{Xu} \mathrm{G}, \mathrm{Li} \mathrm{C}$, et al. IL-17 contributed to the neuropathic pain following peripheral nerve injury by promoting astrocyte proliferation and secretion of proinflammatory cytokines. Molec Med Rep 2017; 15: 89-96. doi: $\quad 10.3892 / \mathrm{mmr} .2016 .6018$. PMID: 27959414.

12. Jafarinia $\mathrm{M}$, Afarideh $\mathrm{M}$, Tafakhori A, Arbabi M, Ghajar A, Noorbala AA, et al. Efficacy and safety of oral ketamine versus diclofenac to alleviate mild to moderate depression in chronic pain patients: A double-blind, randomized, controlled trial. $\boldsymbol{J}$ Affect Dis 2016; 204: 1-8. doi: 10.1016/j.jad.2016.05.076. PMID: 27317968.

13. Zhang M, Harris KM, Ho RC. Is off-label repeat prescription of ketamine as a rapid antidepressant safe? Controversies, ethical concerns, and legal implications. BMC Med Ethics 2016; 117: 4. doi: 10.1186/s12910-016-0087-3. PMID: 26768892.

14. Bayram B, Sayin E, Bozari S, Sahin FM. 2014. HLA-B27 allele frequency in a Turkish study population with primary osteoarthritis. J Rheum 2014; 3: 1-3 doi: 10.4172/2167-6801. 1000117.

15. Rothschild BM. Return to clinically-driven practice, in contrast to serologically-based diagnoses. World J Rheum 2016; 6(1): 1-8. doi: 10.5499/wjr.v6.i1. 1.

16. Rothschild BM, Poteat GB Williams E, Crawford WL. Inflammatory sacroiliac joint pathology: Evaluation of radiologic assessment techniques. Clin Exp Rheum 1994; 12: 267 74.

17. van Onna $\mathbf{M}$, van Tubergen $\mathrm{A}$, van der Heijde DM, Jurik AG, Landewé R. Bone marrow edema on magnetic resonance imaging (MRI) of the sacroiliac joints is associated with development of fatty lesions on MRI over a 1year interval in patients with early inflammatory low back pain: a 2year followup study. J Rheum 2014; 41(6): 1088-94. doi: 10.3899/jrheum.131022.

18. van Tulder MW, Scholten RJ, Koes BW, Deyo RA. Nonsteroidal anti-inflammatory drugs for low back pain: a systematic review within the framework of the Cochrane Collaboration Back Review Group. Spine 2000; 25: 2501-13. PMID: 11013503. doi:10.1097/ 00007632-200010010-00013. 